\title{
CORRECTION
}

\section{Author Correction: Cryo-EM structures of PAC1 receptor reveal ligand binding mechanism}

Jia Wang (D), Xianqiang Song, Dandan Zhang, Xiaoqing Chen, Xun Li, Yaping Sun, Cui Li, Yunpeng Song, Yao Ding, Ruobing Ren, Essa Hu Harrington, Liaoyuan A. Hu (D), Wenge Zhong, Cen Xu, Xin Huang (D, Hong-Wei Wang (D) and Yingli Ma (D)

Cell Research (2020) 30:455; https://doi.org/10.1038/s41422-020-0307-8

Correction to: Cell Research 2020 https://doi.org/10.1038/s41422020-0280-2, published online 11 February 2020

We noticed the missing structure deposition information in our manuscript published online on February 11, 2020, which are the PDB codes and EMDB map entries of the structure
PACAP38-PAC1R-Gs (PDB code: 6M1I and EMDB entry: EMD-30048) and maxadilan-PAC1R-Gs (PDB code: $6 \mathrm{M} 1 \mathrm{H}$ and EMDB entry: EMD-30047). We apologize for any inconvenience this missing information has caused. This correction does not affect the description of the results or the conclusion of this work. 